\title{
An improved model for tidally modulated grounding-line migration
}

\author{
Victor C. TSAI, ${ }^{1}$ G. Hilmar GUDMUNDSSON ${ }^{2}$ \\ ${ }^{1}$ Seismological Laboratory, California Institute of Technology, Pasadena, CA, USA \\ ${ }^{2}$ British Antarctic Survey, Natural Environment Research Council, Cambridge, UK \\ Correspondence: Victor C. Tsai<tsai@caltech.edu>
}

\begin{abstract}
Understanding grounding-line dynamics is necessary for predictions of long-term ice-sheet stability. However, despite growing observations of the tidal influence on grounding-line migration, this short-timescale migration is poorly understood, with most modeling attempts assuming beam theory to calculate displacements. Here we present an improved model of tidal grounding-line migration that treats migration as an elastic fracture problem, forced by the additional ocean water pressure from the tide. This new model predicts that the grounding line cannot be assumed to be in hydrostatic equilibrium and, furthermore, that migration is inherently asymmetric and nonlinear, with migration distances that are not proportional to the tidal load. Specifically, for constant surface slope, the grounding line migrates upstream approximately ten times further as the tide rises from mean sea level to high tide than it migrates downstream as the tide falls from mean sea level to low tide, and migration distances are substantially larger than simple flotation arguments suggest. Numerical tests also show that the dependence of migration distance on elastic moduli and ice-sheet thickness are inconsistent with predictions of beam theory for a range of realistic values. Finally, applying the new model to observations in Antarctica results in new estimates of bed slopes, though these estimates remain uncertain due to imperfect knowledge of the relevant rheological parameters.
\end{abstract}

KEYWORDS: Antarctic glaciology, glacier mechanics, glacier modelling, ice/ocean interactions

\section{INTRODUCTION}

The grounding line marks the division between the grounded and floating parts of an ice sheet. Knowing the position of the grounding line is important for a number of reasons. For example, global mass balance (or rather imbalance) of ice sheets can be estimated by subtracting mass flux across the grounding line from the integrated surface mass balance of the upstream catchment area. The calculated imbalance is often the numerical difference between two much larger numbers, and highly sensitive to any errors in the estimated position of the grounding line (e.g. Shepherd and others, 2012). Accurate data on grounding-line position are also required for any quantitative modeling work aimed at understanding and quantifying the drivers of grounding-line migration and the mechanical interaction between ice shelves and ice sheets (e.g. Schoof, 2007).

Changes in the position of the grounding line may reflect ongoing secular changes in ice geometry or long-term changes in ice-shelf buttressing (e.g. Favier and others, 2014; Joughin and others, 2014; Rignot and others, 2014). The grounding line can also move back and forth with the tide, and tides can therefore serve as a natural experiment to study the mechanical interaction between ice shelves and ice sheets (Brunt and others, 2011). Interestingly, tides have also been found to affect the flow of ice streams tens of kilometers upstream of the grounding line, i.e. far further upstream than the range of tidal flexure (Anandakrishnan and Alley, 1997; Bindschadler and others, 2003; Gudmundsson, 2006). This discovery has led to a renewed interest in understanding the effects of tides on ice flow (Rosier and others, 2014; Thompson and others, 2014).

Tidally driven grounding-line dynamics have been described in a number of different ways. Starting with
Holdsworth (1969, 1977), tidally induced flexure curves around the grounding line have been modeled using simple beam models with a fixed grounding-line position. Walker and others (2013) modeled flexure of an elastic beam on an elastic foundation. This approach allowed them to include deformation upstream of the grounding line. However, they assumed no vertical displacement at the grounding line, i.e. the grounding line acts as a fulcrum. This assumption, which eliminates the possibility of any grounding-line motion, is unlikely to exist in reality, because this would imply the grounding line supports large forces both in vertical tension and compression, neither of which is possible given the known properties of ice (Reeh, 1978; Rist and others, 1999; Reeh and others, 2003).

Sayag and Worster $(2011,2013)$ used a beam model with a migrating grounding line to model tidal flexure. Their model allowed for the movement of the grounding line. In their comparison between their model and data (Sayag and Worster, 2013), however, they used multiple fitting parameters to obtain satisfactory fits to the observed flexure data. Given that the shift in grounding-line position depends strongly on the (generally poorly known) shape of the bedrock, this is a sensible practical approach, but the need for many fitting parameters means that the modeled migration may not be realistic.

One of the key assumptions made in a number of studies of grounding-line dynamics is that the ice directly downstream of the grounding line is in hydrostatic equilibrium. This assumption is made in all vertically integrated largescale ice-flow models, and has also been made in a number of recent studies of tidally induced motion of ice streams (e.g. Bindschadler and others, 2003; Goldberg and others, 2014). Although it seems plausible that the errors in 
calculated grounding-line positions introduced by this assumption will be no larger than a few ice thicknesses, the effects of these errors on grounding-line motion have not been quantified. Comparisons of numerical work using the full-Stokes system (FS, which does not use the hydrostatic assumption), and the shallow ice-stream approximation (SSA, which does use the hydrostatic assumption), suggest that the grounding-line position is located at a greater depth in SSA models than in FS models (Gudmundsson and others, 2012; Pattyn and others, 2013).

Here we propose a new model for tidally modulated grounding-line motion. We treat the grounding-line migration over a tidal cycle as an elastic fracture problem and we formulate and solve a problem of elastic crack growth using a well-established criterion for fracture. In essence, we are viewing the interface between the ice and the bed as a horizontal fracture that opens at the ice-shelf front and extends to a tip at the grounding line, defined by where the ice rests on the bed without an intervening layer of sea water. This approach is fundamentally different from most of the previous work on tidal flexure, which has been based on beam theory. Like beam-theory descriptions, though, our approach also does not rely on the assumption of hydrostatic equilibrium, and we are therefore able to quantify errors in calculated grounding-line motion due to that assumption.

We show that the back-and-forth motion of the grounding line in response to tide is, in general, an inherently asymmetrical problem, and that the inland migration from equilibrium to high tide is expected to be different from that of low tide to equilibrium. To some degree, this is a simple consequence of the fact that downstream of the equilibrium grounding line the upper and the lower surface profiles are related (e.g. in the hydrostatic limit, one can be calculated directly from the other), whereas upstream of the grounding line they are not. Partly, though, the asymmetry is due to the nonlinear relationship between pressure forcing and fracture growth.

\section{ASSUMPTIONS AND FLOTATION HEURISTICS}

The aim of this paper is simply to determine how far the grounding line migrates under tidal forcing in the simple case of a flowline (one-horizontal-dimension) model. Before describing and justifying our model in full, it is worth considering the main physical principles that govern our solution. First, we make the simplifying assumption that only elastic stresses are important over the 12 hour timescales of interest. This will be justified later, but can be understood intuitively as a consequence of there not being sufficient time for significant viscous ice deformation to occur. The second assumption we make is that the ice deforms elastically in response to 'excess' water pressures at the ice/water interface, caused by the difference between hydrostatic water pressure and hydrostatic ice pressure, and that prior to loading, the water and ice pressures were in hydrostatic balance. This hydrostatic assumption may be reasonable for the 12 hour tidal load, since it is expected that water can quickly flow to accommodate deformation over these timescales and grounding-line geometries. The degree to which neglecting the hydrodynamics of water flow is justified can be estimated as follows: Given maximum migration distances of $10 \mathrm{~km}$, fluid velocities of $\sim 0.2 \mathrm{~m} \mathrm{~s}^{-1}$ are needed to fill any void created. But with tidal motions of $\sim 1 \mathrm{~m}$, turbulent flow velocities up to $\sim 0.4 \mathrm{~m} \mathrm{~s}^{-1}$ can be accommodated (Tsai and Rice, 2010). Thus, even in cases where the migration distance is quite large, it is expected that hydrodynamics is only of second order.

Note that although we refer here to the 'hydrostatic assumption', we are not implying that the state of stress within the ice is hydrostatic. The hydrostatic assumption here means that where the ice is afloat, it sinks to a depth where the weight of the ice equals the weight of the ocean column, i.e. $\rho_{\mathrm{i}} g H=\rho g d$, where $\rho_{\mathrm{i}}$ and $\rho$ are the ice and ocean densities, respectively, $g$ is the acceleration due to gravity, $H$ is the ice thickness and $d$ is the draft. This will hold if, for example, vertical stresses within the ice are equal to $\rho_{\mathrm{i}} g$ times depth, which can be referred to as a cryostatic stress state. This is the state of stress if horizontal gradients in shear stress are small compared with vertical gradients in vertical stress. In most approximations used to describe large-scale ice flow (e.g. both the SSA and shallow ice-sheet approximation (SIA)), this holds and the stress state is therefore cryostatic. Our modeling approach does not assume the stress within the ice is either hydrostatic or cryostatic. Here the position of the grounding line is determined by a fracture criterion (given below) and the state of stress will be similar to that obtained by solving the full equilibrium equations. The grounding-line location that fulfills our fracture criterion will, in general, not be the same as the position obtained from the type of hydrostatic considerations employed in most ice-flow models today.

If there were no elastic stresses in the ice, but hydrostatic assumptions still applied, the amount of grounding-line migration would be determined simply by flotation conditions, as is often assumed to be the case in large-scale icesheet models. That is, with no elastic stresses, hydrostatic arguments would dictate that upstream of the grounding line, ice pressure is in excess of the water pressure and downstream of the grounding line, ice pressure is equal to the water pressure on the bottom face of the ice shelf. Assuming a constant surface slope $\alpha$ and bed slope $\beta$ (sloping down towards the coast; Fig. 1a) at the grounding line, hydrostatic for positive tidal amplitude $\Delta h^{+}$(i.e. from equilibrium to high tide), implies

$$
(\alpha-\beta) \rho_{\mathrm{i}} \Delta L^{+}=\rho \Delta h^{+}-\beta \rho \Delta L^{+},
$$

where $\Delta L^{+}$is the upstream migration distance, which can be rewritten as $\Delta L^{+}=\Delta h^{+} / \gamma^{+}$, where

$$
\gamma^{+}=\beta+\frac{\rho_{\mathrm{i}}}{\rho}(\alpha-\beta)=\frac{\rho_{\mathrm{i}}}{\rho} \alpha+\left(1-\frac{\rho_{\mathrm{i}}}{\rho}\right) \beta .
$$

The hydrostatic assumption for negative tidal amplitude $\Delta h^{-}$(i.e. from equilibrium to low tide) implies

$$
\left(1+\frac{\rho_{\mathrm{i}} / \rho}{1-\rho_{\mathrm{i}} / \rho}\right) \alpha \rho_{\mathrm{i}} \Delta L^{-}=\rho \Delta h^{-}-\rho \beta \Delta L^{-},
$$

or $\Delta L^{-}=\Delta h^{-} / \gamma^{-}$, where $\gamma^{-}=\gamma^{+} /\left(1-\rho_{\mathrm{i}} / \rho\right) \approx 9 \gamma^{+}$and $\Delta L^{-}$is the downstream migration distance. Thus, even without elastic effects, tidally modulated grounding-line migration is expected to be asymmetrical, with nine to ten times larger migration upstream than downstream (from equilibrium) for the same tidal amplitude, average surface slope and average bed slope. In other words, we find that the horizontal shift in grounding-line position at high tide, as measured from the position at zero/neutral tide, is not equal in magnitude to that at low tide, and we define this as an asymmetrical tidal response. The asymmetry between high tide and low tide does not imply that the grounding-line 
.

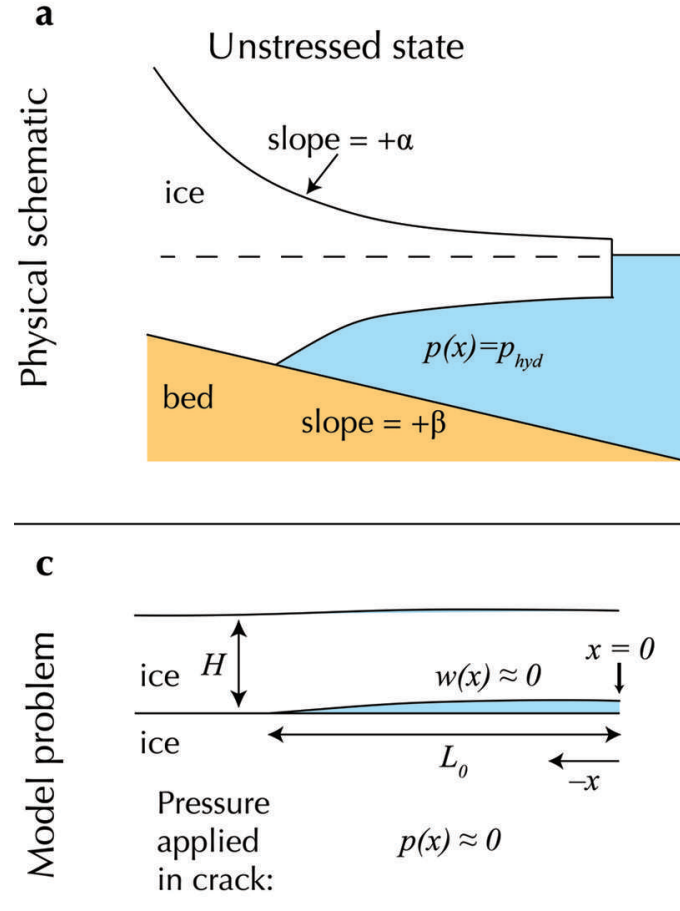

b
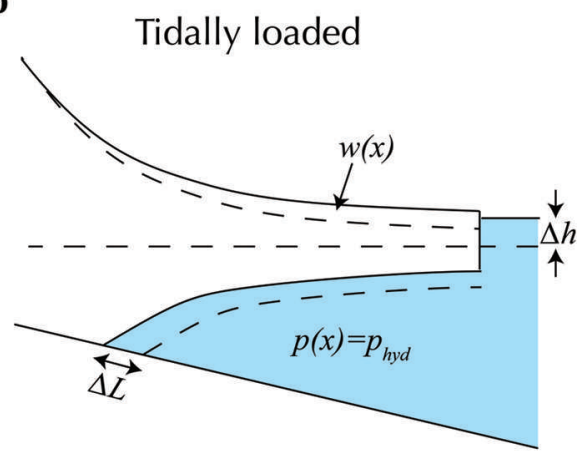

d

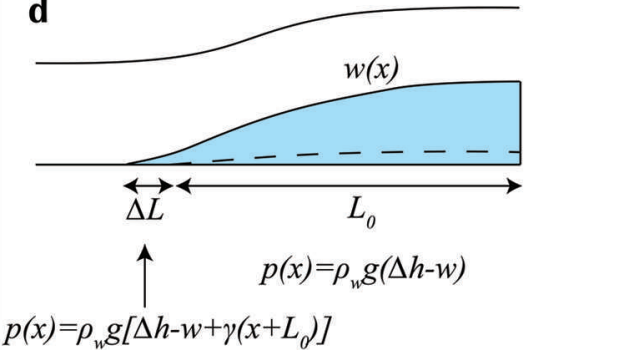

Fig. 1. (a, b) Schematic geometry under (a) unstressed and (b) tidally loaded cases. Water pressure, $p$, is assumed hydrostatic, $p(x)=p_{\text {hyd }}$ and both surface slope and bed slope are assumed constant near the grounding line. (c, d) Modeled crack-opening problem corresponding to the (c) unstressed and (d) tidally loaded cases.

motion does not recover its original position through a complete tidal cycle. In both the hydrostatic model considered here and the elastic model below, the position of the grounding line is the same for the same tidal height, whether the tide is rising or falling.

While the average surface and bed slopes are potentially different immediately upstream versus immediately downstream of the grounding line, the differences are unlikely ever to be $\sim 10$ times different, so this result suggests that grounding-line migration over the positive part of the tidal cycle (high tide) dominates the migration over the negative part of the tidal cycle (low tide). Here we therefore concentrate entirely on describing migration over positive tidal amplitudes. In the hydrostatic limit, we note that this asymmetry always exists provided that $\mathrm{d} H / \mathrm{d} x$ (the ice thickness gradient) is not constant across the grounding line. In particular, if the surface slope is constant across the grounding line then hydrostatic conditions would imply that the thickness gradient is not constant (see Fig. 1a) and hence that the migration is asymmetric. Our general conclusion of asymmetry is different from the symmetric migration of Sayag and Worster (2011), because their study assumes ice of constant thickness across the grounding line, and hence satisfies the special condition that the thickness gradient is constant.

\section{MODEL DESCRIPTION}

\section{Governing equations}

In this model of grounding-line migration over tidal periods ( 1 day), we start by assuming purely elastic ice deformation. Ice is well known to be a nonlinear viscoelastic material (Budd and Jacka, 1989), and it is straightforward to calculate the Maxwell time, which is the timescale over which the deformation transitions from being well approximated as being purely elastic to purely (nonlinear) viscous. For a nonlinear viscous deformation with Glen's flow law exponent $n=3$, rate factor $A=3 \times 10^{-25} \mathrm{~s}^{-1} \mathrm{~Pa}^{-3}$ (Paterson, 1994) and effective Young's modulus $E \approx 10^{9} \mathrm{~Pa}$ (Reeh and others, 2003), an estimate of this Maxwell time is $\sim \eta / E \approx 3 \times 10^{5} \mathrm{~s}$, $\sim 4$ days at an effective stress of $100 \mathrm{kPa}$. Thus, over forcings with periods $<4$ days and stresses of less than $\sim 100 \mathrm{kPa}$, purely elastic models should be a reasonable approximation. In particular, tidal forcings (period 1 day) over ice plains or ice streams (stresses $30 \mathrm{kPa}$ ) should be well approximated by elastic models (Gudmundsson, 2007).

We further assume constant surface slope $\alpha(\mathrm{d} s / \mathrm{d} x=-\alpha)$ and bed slope $\beta(\mathrm{d} b / \mathrm{d} x=-\beta)$ near the grounding line, and simplify the grounding-line geometry to that of a twodimensional plane strain crack, with the crack tip denoting the grounding line (Fig. 1a), homogeneous ice of elastic modulus $E^{\prime}=E /\left(1-\nu^{2}\right)=2 \times 10^{9} \mathrm{~Pa}$, where $\nu$ is Poisson's ratio, and water at hydrostatic pressure filling the crack. As discussed above, it is expected that grounding-line migration is $\sim 10$ times larger over positive forcing (i.e. crack opening from equilibrium) compared with negative forcing (i.e. crack closure from equilibrium). This implies that the migration calculated from equilibrium to high tide is accurate to within $10 \%$ of the total migration (from low tide to high tide). For the remainder of this work, we therefore only treat the positive forcing case, where the crack is expected to grow by a positive value, $\Delta L>0$. It is worth noting that the zero-stress, equilibrium reference point cannot be chosen arbitrarily. For example, one cannot choose the low tide as the reference point from which crack growth occurs because this low tide configuration is not an unstressed state. As discussed in more detail below, grounding-line migration is an inherently nonlinear problem.

To account for the pre-tidally stressed geometry (which is set by viscous deformation), for the unstressed state, we 
assume zero elastic crack opening and zero excess pressure over the unstressed crack. Over the tidally stressed crack, we assume that the pressure over the original crack face is the hydrostatic pressure difference caused by the difference between the new crack opening, $w$, and the tidal forcing, $\Delta h$, whereas the pressure over the newly created crack face is this same hydrostatic pressure difference plus the hydrostatic adjustment due to bed slope (see Fig. 1b). (Note that the crack face can be interpreted as the icy roof of the horizontal fracture.) Specifically, within the crack $\left(-L \equiv-L_{0}-\Delta L<x<0\right)$, we set the excess pressure to be

$$
\begin{gathered}
p(x)=\rho g[\Delta h-w(x)],-L_{0}<x<0 \\
p(x)=\rho g\left[\Delta h-w(x)+\gamma\left(x+L_{0}\right)\right],-L_{0}-\Delta L<x<-L_{0}
\end{gathered}
$$

where $\Delta h$ is the tidal height difference, $w(x)$ is the displacement from the unstressed state, $\rho$ is the density of water, and the extended crack is from $x=-L_{0}-\Delta L$ to $x=0$ (with a new grounding line at $x=-L_{0}-\Delta L$ and the iceshelf edge still at $x=0$ ). The $\gamma$ factor accounts for both hydrostatic ice and water pressure variations within the extended part of the crack, and therefore is given by Eqn (2), i.e. $\gamma=\gamma^{+}$. For simplicity, we assume constant ice thickness $H$. While this may be a crude assumption in some cases, the general behavior of the final solution is not expected to be significantly different, as long as $H$ thins moderately slowly, which is the case for many Antarctic ice streams and ice plains. Finally, we assume that $w \ll L_{0}$, such that linear elasticity applies.

To complete the description of the elastic crack problem, we need to provide conditions for crack growth and conditions at $x=0$. Since the fracture toughness of ice $\left(K_{\mathrm{IC}} \sim 150 \mathrm{kPa} \mathrm{m}^{1 / 2}\right.$; Rist and others, 1999) is small compared with $\rho g \Delta h \sqrt{L_{0}} \approx 600 \mathrm{kPam}^{1 / 2}$ for $\Delta h>2 \mathrm{~m}$ and $L_{0}>1 \mathrm{~km}$, and the fracture toughness of the ice/till/bedrock interface is expected to be even lower than that of pure ice, we assume the fracture toughness is approximately zero, so there is no resistance to crack opening. Note that this is in contrast to the fulcrum assumption of Walker and others (2013), which would require a much larger fracture toughness than is observed to accommodate the large stresses that would result in such a case. Our fracture criterion is then

$$
K=K_{\mathrm{IC}}=0,
$$

where $K$ is the stress intensity factor. For an ice shelf that is significantly longer than the flexural length scale, elastic stresses within the ice shelf are expected to decay, so that stresses are nearly zero at $x=0$. For this case, the problem to be solved is equivalent to a crack of length $2\left(L_{0}+\Delta L\right)$ that is symmetric with respect to $x=0$. We assume this is the case. It may be noted that this symmetry assumption is not a limitation of the model, since the bending stresses in our solutions at $x=0$ are negligible (for long enough $L_{0}$ ), implying that the symmetric assumption is virtually identical to the more realistic free ice face condition.

With the previous assumptions, the elastic crack equations are well posed and can be written as a set of two coupled equations (Tsai and Rice, 2012)

$$
\begin{array}{r}
0=-\sigma_{x z}(x)=\int_{-L_{0}-\Delta L}^{L_{0}+\Delta L}\left[\left(\frac{1}{s-x}+k_{11}\right) \frac{\partial u}{\partial s}+k_{12} \frac{\partial w}{\partial s}\right] \mathrm{d} s, \\
-\frac{4 \pi p(x)}{E^{\prime}}=\int_{-L_{0}-\Delta L}^{L_{0}+\Delta L}\left[k_{21} \frac{\partial u}{\partial s}+\left(\frac{1}{s-x}+k_{22}\right) \frac{\partial w}{\partial s}\right] \mathrm{d} s,
\end{array}
$$

along with the fracture criterion of Eqn (5). Here the $k_{i j}=k_{i j}(x, s ; H)$ are the elasticity kernels, as in Tsai and Rice (2012) (which depend on $H$ ), and Eqns (6) describe the relationship between shear stress $\sigma_{x z}(x)$ and pressure $p(x)$ along the crack, and crack-opening displacements $u(x)$ and $w(x)$ (horizontal and vertical displacement, respectively).

Finally, although not realistic for ice shelves, it is worth noting that when $H \gg L_{0}, k_{i j}=0, u(x)=0$, and the three governing equations of Eqns (5) and (6) simplify to two equations

$$
K=\int_{-L_{0}-\Delta L}^{L_{0}+\Delta L} \frac{p(x) \mathrm{d} x}{\sqrt{\left(L_{0}+\Delta L\right)^{2}-x^{2}}}=0
$$

and

$$
\frac{4 \pi p(x)}{E^{\prime}}=\int_{-L_{0}-\Delta L}^{L_{0}+\Delta L} \frac{\partial w}{\partial s} \frac{\mathrm{d} s}{x-s},
$$

as in Tsai and Rice (2010). For this simplified model (which could be applied to a tidewater glacier without an ice shelf), it can be observed that Eqn (7) implies that $p(x)$ must take both positive and negative values in order to be satisfied. Since $p(x)$ is expected to be positive over the original crack face (for a positive $\Delta h$ ) and decrease over the newly formed crack face, it is impossible for the perturbed grounding-line position $x_{\mathrm{GL}}^{\mathrm{p}}=-L_{0}-\Delta L$ to be achieved at local flotation, where $p=0$. In other words, even without performing the full calculation, we can show that an important consequence of the governing equations is that the new grounding-line position $x_{\mathrm{GL}}^{\mathrm{p}}$ will be upstream of the position of local flotation. This is also true of the nonsimplified Eqn (5), and is discussed in more detail in the following sections.

\section{Numerical method}

For given values of $\Delta h, \gamma, L_{0}, H / L$ and $E^{\prime}$ (hereafter called 'fixed parameters'), Eqns (4-6) can be solved uniquely for $p(x), w(x), u(x)$ and $\Delta L$. To numerically obtain a solution, we follow the approach of Tsai and Rice (2012) and use the Chebyshev method of Erdogan and others (1973) to solve Eqns (6) for $w(x)$ and $u(x)$ for a given $p(x)$ and $\sigma_{x z}=0$. For given values of the fixed parameters, and a choice of $\Delta L$, a nonlinear least-squares routine is used to minimize the difference between the pressure $p(x)$ as calculated from Eqn (6b) and that calculated from Eqn (4). This solution for $p(x)$ and $w(x)$ generally does not satisfy the fracture criterion of Eqn (5), and we use a secant method to iteratively solve for the $\Delta L$ that satisfies Eqn (5). For all calculations shown, we use a 35-term Chebyshev series to approximate $p(x)$ and $w(x)$, and a 45-point Chebyshev-Gauss quadrature to evaluate integrals.

\section{NUMERICAL RESULTS}

In Figure 2, we show the excess pressure profile $p(x)$ and the opening profile $w(x)$ for a representative set of fixed parameters, $\Delta h=2 \mathrm{~m}, \gamma=0.001, L_{0}=10 \mathrm{~km}, H=1 \mathrm{~km}$, $E^{\prime}=2 \times 10^{9} \mathrm{~Pa}$. First, we observe that for this case, $\Delta L \approx 3.71 \mathrm{~km}$, so the new crack length is $13.7 \mathrm{~km}$. For a given $\Delta h$ and $\gamma$, one can calculate the migration expected to maintain flotation at the grounding line, $\Delta L_{\mathrm{fl}} \equiv \Delta h / \gamma$. In this case, then, $\Delta L_{\mathrm{fl}}=2 \mathrm{~km}$. Thus, as pointed out above, the grounding line migrates substantially further over a tidal cycle than is predicted by a simple flotation argument, 


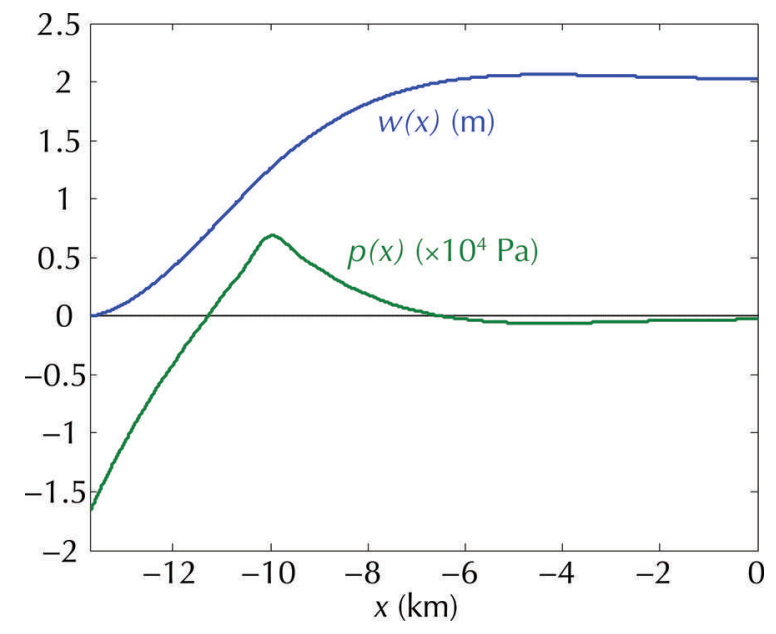

Fig. 2. Excess pressure, $p(x)$ (green), and opening profile, $w(x)$ (blue).

because of the elastic stresses that exist within the ice. Also, one may note that $w(0)=\Delta h$, so that $p(0)=0$, which are both expected when $L_{0}$ is long enough. It is also worth noting that the opening profile, $w(x)$, is consistent with the beam-theory approximation that would have been produced for the same pressure profile, $p(x)$, for regions sufficiently far away from the grounding line (crack tip), explaining why beam-theory models have produced reasonable fits to observed ice-shelf flexure profiles. However, close to the grounding line (i.e. within a few ice thicknesses), our profile is different from the beam-theory approximation and data from this region could be used to distinguish the present fully elastic model from approximate beam-theory models. Finally, we note that there is a small numerical artifact (slight smoothing of the pressure profile) near the original crack tip location, $x=-10 \mathrm{~km}$, due to the truncation of terms in the Chebyshev series, which could be lessened by taking more terms in the series. Since we do not aim to interpret any results to a high degree of accuracy $(<1 \%)$, we ignore such features and do not discuss them further.

To show how changes to individual fixed parameters affect $\Delta L$, in Figure 3 we show parametric studies, where we systematically vary $L_{0}, E^{\prime}, H$ and $\Delta h / \gamma$. For this study, we maintain constant values of all other fixed parameters and only vary the parameter in question. For variable $L_{0}$ (Fig. 3a), we observe two notable features. First, for $L_{0} \gtrsim 5 \mathrm{~km}$, $\Delta L \approx 3.7 \mathrm{~km}$, i.e. $\Delta L$ is independent of $L_{0}$. This is expected, since ice-shelf flexure can accommodate $w(x) \approx \Delta h$ and $p(x)=0$ for $x$ sufficiently downstream of the grounding line. The second notable feature is the limit of $L_{0}=0$. In this case, $\Delta L / \Delta L_{\mathrm{fl}}=1.57$. Examination of the $p(x)$ profile shows that $p(x)$ is nearly linear over the entire crack, i.e. $p(x) \approx a_{0}\left(x-x_{0}\right)$. Since $H / L \gg 1$, Eqn (7) applies (with $\left.L_{0}=0\right)$, and substituting the linear profile for $p(x)$ results in $x_{0}=-2 / \pi$. In other words, $\Delta L / \Delta L_{\mathrm{fl}}=\pi / 2 \approx 1.57$, which is numerically verified. Finally, it may be observed that with $L_{0}$
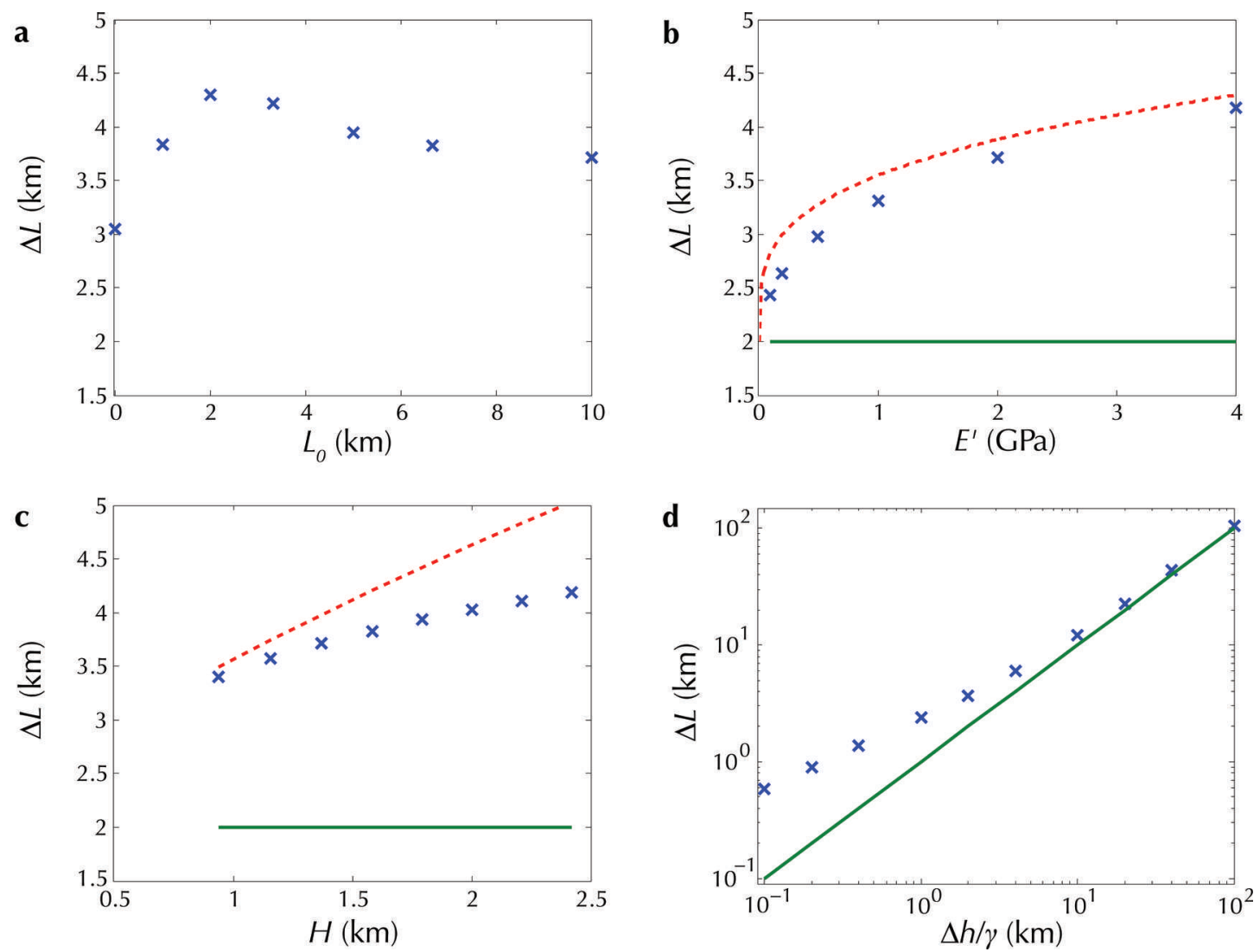

Fig. 3. Grounding-line migration, $\Delta L$, as a function of (a) $L_{0}$, (b) $E^{\prime}$, (c) $H$ and (d) $\Delta h / \gamma$, where other parameters are kept constant. Blue crosses are model results, and the green line denotes $\Delta L_{\mathrm{fl}}$, the value of $\Delta L$ at flotation. When not varied, the default values of parameters chosen here are $\Delta h=4 \mathrm{~m}, \gamma=2 \times 10^{-3}, L_{0}=10 \mathrm{~km}, H / L=0.1$ and $E^{\prime}=2 \mathrm{GPa}$. In (a) $H / L \times L_{0}$ is kept at a constant value of 0.1 , except when $L_{0}=0$, where $H / L=100$. The red dashed curves in (b) and (c) denote the scaling expected of beam theory (e.g. Holdsworth, 1969; Sayag and Worster, 2011) added to the hydrostatic migration. (c) is calculated by fixing $H / L=0.1$ and varying $L_{0}$ from 6 to $20 \mathrm{~km}$. Since $L_{0} \gtrsim 5 \mathrm{~km}$, it is expected that the only effect of changing $L_{0}$ is changing $H$. (d) shows that $\Delta L$ is nonlinearly related to $\Delta h$. 
fixed (e.g. to a value $>5 \mathrm{~km}$ ), our model has four remaining parameters $\left(E^{\prime}, H, \Delta h\right.$ and $\left.\gamma\right)$.

Figure $3 \mathrm{~b}$ shows the dependence of $\Delta L$ on $E^{\prime}$. The general form of the dependence is as expected, with $\Delta L$ increasing with $E^{\prime}$, from an expected value of $\Delta L \approx \Delta L_{\mathrm{fl}}$ at $E^{\prime}=0$. Similarly, the increase of $\Delta L$ with $H$ (Fig. 3c) is expected, since larger values of $H$ result in a stiffer overall system. For example, in the limit of beam theory, the effective stiffness is proportional to $H^{3} E^{\prime}$ (Holdsworth, 1969; Vaughan, 1995). However, it should be observed that the dependence of $\Delta L$ on $E^{\prime}$ and $H$ does not follow the scaling expected of beam theory, $\Delta L \propto\left(H^{3} E^{\prime}\right)^{1 / 4}$ (red curves in Fig. 3b and c, which denote the hydrostatic prediction added to the beam-theory scaling with an arbitrary scale factor). Importantly, this suggests that calculations of grounding-line behavior utilizing beam theory are not quantitatively accurate over a range of parameter space. Specifically, although beam theory may fit tidal flexure observations reasonably well (as mentioned above), the predicted grounding-line migration distances are somewhat different for the realistic parameter choices shown in Figure 3. It is also interesting to note that the beam-theory results of Sayag and Worster (2011) agree well with their 'constant-thickness' deformation experiments, and that this agreement implies that our results are also inconsistent with the laboratory experiments Sayag and Worster (2011) used to test the appropriateness of their beam-theory calculations. While a complete understanding of why this inconsistency exists is beyond the scope of this paper, we provide one possible reason for this. Perhaps the most important difference is that the experiments of Sayag and Worster (2011) (as well as their beam-theory numerical calculations) are performed without first achieving a state of equilibrium stress, where all stresses within the elastic sheet would be zero, whereas our calculations explicitly assume there exists a state of equilibrium stress at the equilibrium tide reference state, as expected of a real ice shelf, given that long-term viscous ice deformation should relax stresses to zero for equilibrium tidal conditions (over long enough time). Thus, the experiments and calculations of Sayag and Worster (2011) may not be a good approximation of the real grounding-line problem, where there is long-term viscous deformation.

For the dependence of $\Delta L$ on $\Delta h$ and $\gamma$, we first note that a simplification can be made if we can rewrite the governing equations in non-dimensional form, where $\widehat{p}=p /\left(\gamma E^{\prime}\right)$, $\widehat{w}=w / \Delta h$ and $\widehat{x}=\gamma x / \Delta h$. With this substitution, it is clear that the governing equations only contain $\Delta h$ and $\gamma$ as the ratio, $\Delta h / \gamma$. Thus, the solution (including $\Delta L$ ) is affected equally by changes in $\Delta h$ and $\gamma^{-1}$. Figure $3 d$ shows the dependence of $\Delta L$ on $\Delta h / \gamma$. As shown, $\Delta L>\Delta L_{\mathrm{fl}} \equiv \Delta h / \gamma$ for all values of $\Delta h / \gamma$, with $\Delta L / \Delta L_{\mathrm{fl}} \rightarrow 1$ as $\Delta h / \gamma \rightarrow \infty$, and the ratio growing as $\Delta h / \gamma \rightarrow 0$. Despite the large values of $\Delta L / \Delta L_{\mathrm{fl}}$, the absolute value of $\Delta L \rightarrow 0$ as $\Delta h / \gamma \rightarrow 0$, as expected. It is of interest to point out that the nonlinearity of $\Delta L$ vs $\Delta h / \gamma$ implies that grounding-line migration is not equal for each increment of forcing, $\Delta h$. This nonlinear dependence of grounding-line migration on tidal forcing highlights the asymmetry inherent in grounding-line migration, which has also been seen in recent full viscoelastic modeling of grounding-line migration (Rosier and others, 2014), and also implies that the solution depends on the (zero stress) reference state. Unlike linear problems where the reference state can be chosen arbitrarily, grounding-line tidal migration is sensitive to what the true unstressed reference state is.

\section{ESTIMATING BED SLOPE}

We provide here one simple application of the modeling framework presented above in estimating bedrock slopes using tidal observations. Brunt and others (2011) used satellite observations of grounding-line migration to estimate bed slopes at a few ice plains of the Filchner-Ronne Ice Shelf, West Antarctica, based on a hydrostatic argument. Our results suggest that these estimates can be improved in two ways.

First, even for hydrostatic migration, Eqns (2) and (3) suggest that the full tidal migration slope, $\Delta h / \Delta L$ (full tidal amplitude divided by migration distance), is approximately given by $1.8 \gamma^{+}$. For example, taking at face value the values of $\Delta h / \Delta L=6 \mathrm{~m} / 7000 \mathrm{~m}=8 \times 10^{-4}$ and $\alpha=1 \times 10^{-4}$, measured by Brunt and others (2011) for Bungenstrockrucken Ice Plain, a hydrostatic assumption would imply a local bed slope of $\beta \approx 3 \times 10^{-3}$. This is larger than the value estimated by Brunt and others (2011) of $1-2 \times 10^{-3}$, which is an invalid estimate, partly due to their unjustified assumption that bed slope can be equated to the tidal migration slope.

Inclusion of the elastic stresses further increases the predicted bed slopes. Taking $h \approx 1000 \mathrm{~m}$ from Bedmap2 (Fretwell and others, 2013) and assuming $E^{\prime} \approx 2 \times 10^{9} \mathrm{~Pa}$, Figure $3 \mathrm{~d}$ suggests that $\Delta h^{+} / \gamma^{+} \approx 4 \mathrm{~km}$, or $\gamma^{+} \approx$ $1.75 \Delta h^{+} / \Delta L^{+}$and so $\beta \approx 6 \times 10^{-3}$. While this value of bed slope is uncertain, due to large uncertainties in the parameters used, this comparison demonstrates that the resulting bed slope can be significantly larger than estimates based on hydrostatic assumptions. This result suggests that, at a minimum, care should be taken in using tidal grounding-line migration to quantitatively infer bed slopes. If precise values for bed slope are desired from such an approach, it is necessary to have well-constrained measurements of surface slopes, (effective) elastic moduli, ice thicknesses and grounding-line migration distances.

\section{SUMMARY AND CONCLUSIONS}

We have introduced a new approach to the treatment of grounding-line motion over tidal cycles, based on elastic fracture mechanics. The movement of the grounding line is recast as a crack-growth problem with the grounding line representing the tip of an elastic crack. The equations governing the movement of the grounding line are similar to those controlling the opening and closing of a water-filled crack under pressure.

We show that even when assuming hydrostatic grounding-line migration, the migration is greater from equilibrium to high tide than from low tide to equilibrium (by a factor of ten, for the same surface and bed slopes). This asymmetry is also found to be the case in our more general elastic treatment of this problem, as again we find that the grounding-line migration is nonlinear with respect to tidal forcing. In qualitative (but not quantitative) agreement with beam-theory models, elastic stresses within the ice cause the grounding line to migrate substantially further than a simple flotation argument would suggest. In fact, inspection of the governing equations of the elastic fracture problem already reveals that the grounding-line position at high tide is always upstream of the position of local flotation. Application of this new framework to observations of grounding-line migration therefore results in estimates of bed slopes that are greater than would be predicted by purely hydrostatic migration. The 
new framework also suggests differences in the ice stresses near the grounding line, which has implications for ice-shelf damage, crevassing and ice seismicity, but a quantitative analysis of such effects is left for future work.

Although our analysis here focuses on grounding-line migration in response to tides (and therefore includes elastic stresses only), we suggest that some of our conclusions may also hold for the purely viscous case. In particular, we surmise that our finding that the grounding line is located further upstream when additional stresses beyond the cryostatic stress state are included is not limited to the purely elastic case. If true, this could explain why grounding-line positions in full-Stokes models are generally located upstream of those in SSA models.

\section{ACKNOWLEDGEMENTS}

We thank Roiy Sayag and two anonymous reviewers for constructive reviews, and the California Institute of Technology for supporting G.H.G.'s visit. This research was carried out at the California Institute of Technology and the Jet Propulsion Laboratory under a contract with the National Aeronautics and Space Administration and partly funded through the President's and Director's Fund Program.

\section{REFERENCES}

Anandakrishnan S and Alley RB (1997) Tidal forcing of basal seismicity of Ice Stream C, West Antarctica, observed far inland. J. Geophys. Res., 102(B7), 15 183-15196 (doi: 10.1029/97JB01073)

Bindschadler RA, King MA, Alley RB, Anandakrishnan S and Padman L (2003) Tidally controlled stick-slip discharge of a West Antarctic ice stream. Science, 301(5636), 1087-1089 (doi: 10.1126/science.1087231)

Brunt KM, Fricker HA and Padman L (2011) Analysis of ice plains of the Filchner-Ronne Ice Shelf, Antarctica, using ICESat laser altimetry. J. Glaciol., 57(205), 965-975 (doi: 10.3189/ 002214311798043753)

Budd WF and Jacka TH (1989) A review of ice rheology for ice sheet modelling. Cold Reg. Sci. Technol., 16(2), 107-144 (doi: 10.1016/0165-232X(89)90014-1)

Erdogan F, Gupta GD and Cook TS (1973) Numerical solution of angular integral equations. In Sih GC ed. Methods of analysis and solution of crack problems: recent developments in fracture mechanics. Noordhoff International Publishing, San Diego, CA, and Leyden, 368-425

Favier L and 8 others (2014) Retreat of Pine Island Glacier controlled by marine ice-sheet instability. Nature Climate Change, 4(2), 117-121 (doi: 10.1038/nclimate2094)

Fretwell P and 59 others (2013) Bedmap2: improved ice bed, surface and thickness datasets for Antarctica. Cryosphere, 7(1), 375-393 (doi: 10.5194/tc-7-375-2013)

Goldberg N, Schoof C and Sergienko OV (2014) Stick-slip motion of an Antarctic ice stream: the effects of viscoelasticity. J. Geophys. Res. Earth Surf., 119(7), 1564-1580 (doi: 10.1002/ 2014JF003132)

Gudmundsson $\mathrm{GH}$ (2006) Fortnightly variations in the flow velocity of Rutford Ice Stream, West Antarctica. Nature, 444(7122), 1063-1064 (doi: 10.1038/nature05430)

Gudmundsson GH (2007) Tides and the flow of Rutford Ice Stream, West Antarctica. J. Geophys. Res., 112(F4), F04007 (doi: 10.1029/2006JF000731)
Gudmundsson GH, Krug J, Durand G, Favier L and Gagliardini O (2012) The stability of grounding lines on retrograde slopes. Cryosphere, 6(6), 1497-1505 (doi: 10.5194/tc-6-1497-2012)

Holdsworth G (1969) Flexure of a floating ice tongue. J. Glaciol., 8(54), 385-397

Holdsworth G (1977) Tidal interaction with ice shelves. Ann. Géophys., 33(1/2), 133-146

Joughin I, Smith BE and Medley B (2014) Marine ice sheet collapse potentially under way for the Thwaites Glacier Basin, West Antarctica. Science, 344(6185), 735-738 (doi: 10.1126/ science.1249055)

Paterson WSB (1994) The physics of glaciers, 3rd edn. Elsevier, Oxford

Pattyn F and 27 others (2013) Grounding-line migration in planview marine ice-sheet models: results of the ice2sea MISMIP3d intercomparison. J. Glaciol., 59(215), 410-422 (doi: 10.3189/ 2013JoG12J129)

Reeh N (1978) On the application of beam theory to glacier mechanics. (PhD Thesis, Technical University of Denmark)

Reeh N, Lintz Christensen E, Mayer C and Olesen OB (2003) Tidal bending of glaciers: a linear viscoelastic approach. Ann. Glaciol., 37, 83-89 (doi: 10.3189/172756403781815663)

Rignot E, Mouginot J, Morlighem M, Seroussi H and Scheuchl B (2014) Widespread, rapid grounding line retreat of Pine Island, Thwaites, Smith, and Kohler glaciers, West Antarctica, from 1992 to 2011. Geophys. Res. Lett., 41(10), 3502-3509 (doi: 10.1002/2014GL060140)

Rist MA and 6 others (1999) Experimental and theoretical fracture mechanics applied to Antarctic ice fracture and surface crevassing. J. Geophys. Res., 104(B2), 2973-2987 (doi: 10.1029/1998JB900026)

Rosier SHR, Gudmundsson GH and Green JAM (2014) Insights into ice stream dynamics through modelling their response to tidal forcing. Cryosphere, 8(5), 1763-1775 (doi: 10.5194/tc-81763-2014)

Sayag R and Worster MG (2011) Elastic response of a grounded ice sheet coupled to a floating ice shelf. Phys. Rev. E, 84(3), 036111 (doi: 10.1103/PhysRevE.84.036111)

Sayag R and Worster MG (2013) Elastic dynamics and tidal migration of grounding lines modify subglacial lubrication and melting. Geophys. Res. Lett., 40(22), 5877-5881 (doi: 10.1002/ 2013GL057942)

Schoof C (2007) Marine ice-sheet dynamics. Part 1. The case of rapid sliding. J. Fluid Mech., 573, 27-55 (doi: 10.1017/ S0022112006003570)

Shepherd A and 46 others (2012) A reconciled estimate of ice-sheet mass balance. Science, 338(6111), 1183-1189 (doi: 10.1126/ science.1228102)

Thompson J, Simons M and Tsai VC (2014) Modeling the elastic transmission of tidal stresses to great distances inland in channelized ice streams. Cryosphere, 8(6), 2007-2029 (doi: 10.5194/tc-8-2007-2014)

Tsai VC and Rice JR (2010) A model for turbulent hydraulic fracture and application to crack propagation at glacier beds. J. Geophys. Res., 115(F3), F03007 (doi: 10.1029/ 2009JF001474)

Tsai VC and Rice JR (2012) Modeling turbulent hydraulic fracture near a free surface. J. Appl. Mech., 79(3), 031003 (doi: 10.1115/ 1.4005879)

Vaughan DG (1995) Tidal flexure at ice shelf margins. J. Geophys. Res., 100(B4), 6213-6224 (doi: 10.1029/94JB02467)

Walker RT, Parizek BR, Alley RB, Anandakrishnan S, Riverman KL and Christianson K (2013) Ice-shelf tidal flexure and subglacial pressure variations. Earth Planet. Sci. Lett., 361, 422-428 (doi: 10.1016/j.epsl.2012.11.008) 